\title{
Fast finger design automation for industrial robots
}

Mohammadali Honarpardaz, Johan Andersson (Ölvander) and Mehdi Tarkian

The self-archived postprint version of this journal article is available at Linköping University Institutional Repository (DiVA):

http://urn.kb.se/resolve?urn=urn:nbn:se:liu:diva-153950

N.B.: When citing this work, cite the original publication.

Honarpardaz, M., Andersson (Ölvander), J., Tarkian, M., (2019), Fast finger design automation for industrial robots, Robotics and Autonomous Systems, 113, 120-131.

https://doi.org/10.1016/j.robot.2018.12.011

Original publication available at:

https://doi.org/10.1016/j.robot.2018.12.011

Copyright: Elsevier

http://www.elsevier.com/

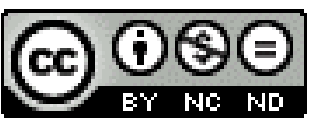




\title{
Agile Finger Design Automation for Industrial Robots
}

\author{
M. Honarpardaz a, b, J. Ölvander ${ }^{\text {b }}$, M. Tarkian ${ }^{\text {b }}$ \\ ${ }^{a}$ ABB, Corporate Research Center, Forskargränd 7, Västerås 721 78, Sweden \\ ${ }^{\mathrm{b}}$ Linköping University, Division of Machine Design, Linköping 581 83, Sweden
}

\section{Corresponding author:}

Mohammadali Honarpardaz, ABB, Corporate Research Center, Forskargränd 7, Västerås 721 78, Sweden.

Email: mohammadali.honarpardaz@se.abb.com

\begin{abstract}
Finger design automation is highly demanded from robot industries to fulfill the requirements of the agile market. Nevertheless, literature lacks a promising approach which automates the design process of reliable fingers for industrial robots. Hence, this work proposes the generic optimized finger design (GOFD) method which automates the design process and minimizes the time it takes to design finger grippers. The method is applied to optimize fingers for several objects and it outperforms existing methods. Finally, the optimized fingers are manufactured and the results are experimentally verified.
\end{abstract}

\section{Keywords}

Optimization, fingers design, design automation, grasp synthesis, industrial grippers, robotics

\section{Introduction}

Fingers are fundamental items of robot manipulators as they are the only interfaces that connect the robot to the outside world. Properly designed fingers play a significant role in increasing the performance and consequently the throughput of workcells. On the other hand, inefficient fingers may lead to failure of the workcell by damaging workpieces and robot hardware. Designing reliable fingers therefore requires excellent skills in robotics as well as mechanical design (Nof, 1999).

Robots are increasingly being used for new applications in markets they have never been used in before. The new trends are especially visible in the area of assembly, where traditionally either low-cost labor or hard automation (i.e. make use of specially designed machines and equipment for production) was used (Siciliano et al., 2010). The complex and long robot integration processes undermine robotics in the current highly competitive 
agile manufacturing where production is ramped up to full volume in a very short time and lasts for 3-12 months. Many robotic industries have targeted enabling non-skilled users to integrate a robot system for an assembly application in a short time frame. SARAFun (SARAFun, 2017) and Factory-in-a-day (Factory-in-a-day, 2017) are two examples of European Commission projects that are investigating different approaches to expedite and simplify the robot integration process.

In assembly applications, a robot arm must be able to handle multiple workpieces to accomplish an assembly. In order to handle multi-objects, various methods and systems are developed (Tai et al., 2016) yet few are embraced by robot industries due to their limitations. One of the most common industrial multi-object grasp systems (MOGS) is multi-function fingers, in which fingers are designed to handle specific objects without any tool-change operation (Greg Causey, 2003). Today, multi-function fingers are manually designed in an iterative and time-consuming trial-and-error process. Therefore, designing functional and efficient fingers takes several weeks even for highly skilled designers. As the current exhaustive manual finger design method makes up a large portion of the integration process, research within automating finger designs has become a hot topic for robot manufacturers.

In earlier works (Honarpardaz et al., 2016a, 2016b), the Generic Automated Finger Design (GAFD) method is introduced to enable a non-skilled user to generate customized fingers for applications that require handling one or more workpieces (e.g. pick-and-place and assembly). The proposed method successfully produces fingers for complex workpieces. Performance and stability of the designed fingers are experimentally verified and compared with existing automated finger design tools (Honarpardaz et al., 2017a). Results show that fingers designed using GAFD have considerably smaller footprints and consequently larger approach spaces in comparison to other methods.

In the GAFD method, a CAD model of the workpiece is discretized to obtain a point cloud model of the workpiece, as demonstrated in Fig. 1 (a) using a battery as an example. An exhaustive search method is then employed to synthesize and analyze grasps based on the point cloud. The algorithm searches through all possible grasp sets and evaluates the quality of each grasp set by using the ball criterion (Ferrari and Canny, 1992). According to this quality metric, the radius $(\varepsilon)$ of the largest ball centered at the origin of a wrench space and inscribed in the convex hull of contact wrenches is considered the grasp quality. All grasp sets are then ranked based on their quality and the best grasp set is selected.

Despite the fact that the proposed method can significantly reduce the design process time (DPT), it is highly dependent on the size of point clouds, which stems from the geometrical complexity and volume of workpieces. As shown in Fig. 1 (b), in some cases where the point cloud models are large, the design time might be up to 25 hours for each workpiece; this method is therefore inapplicable for one-day-integration applications. 
a)

a) Point Cloud Model of an AAA Battery

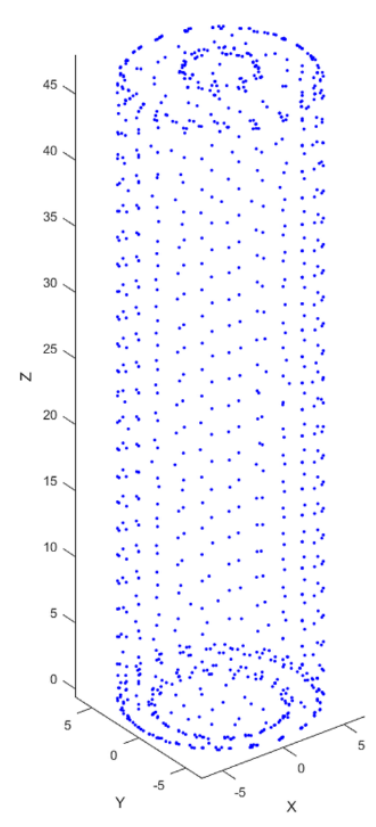

b)

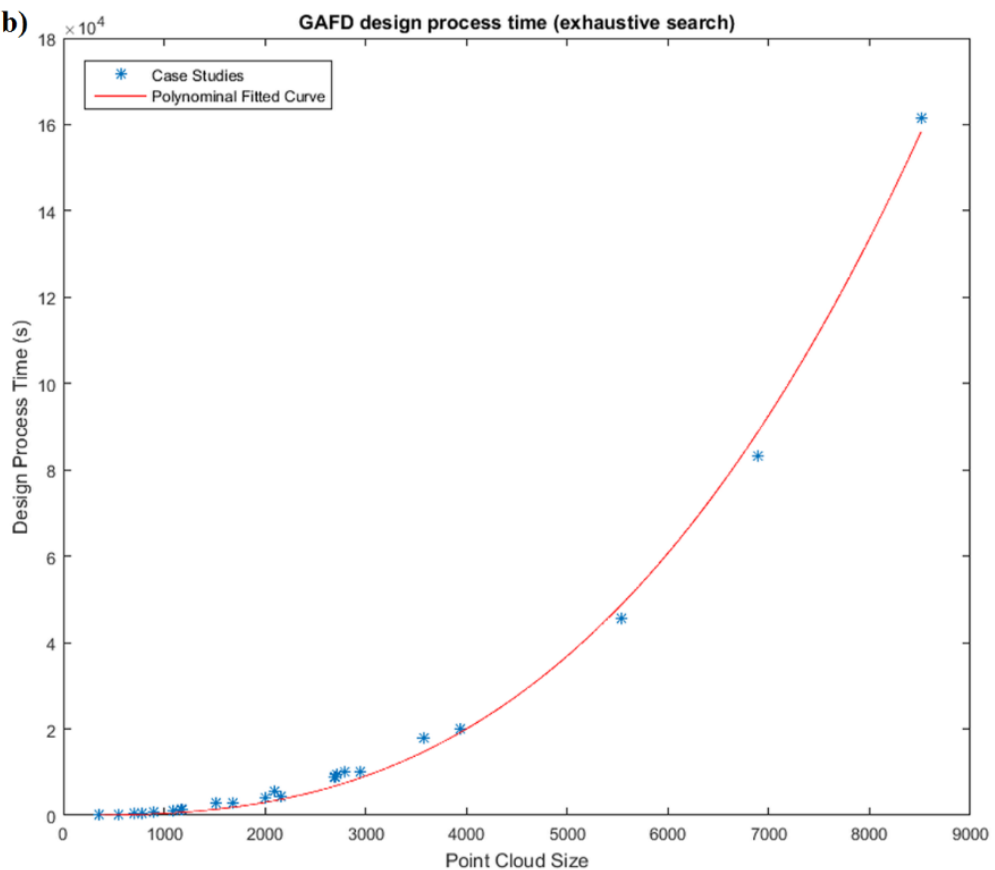

Fig. 1: Examples of: (a) point cloud model of an AAA battery, and (b) the design process time of the GAFD method.

This paper proposes the Generic Optimized Finger Design (GOFD) method as an extension of previously developed methods in order to reduce the design time by introducing a mathematical optimization process to speed up the search without affecting the reliability of the fingers. The remainder of this article is branched into sections as follows. Section 2 presents an overview of multi-object handling methods and relevant works are reviewed in Section 3. Section 4 describes the proposed methodology and the investigated case studies are defined in Section 5. Results are presented in Section 6 and finally extensively discussed and analyzed in Section 7.

\section{Background}

Handling multiple objects has always been a major challenge in robotics, yet in the last decade research on finding a generic system has increased considerably (Kawasaki, 2015). Multi-object grasp systems (MOGS) are developed to enable robots to grasp and manipulate objects of various shapes and sizes. Studies in the MOGS research field may be categorized into anthropomorphic hands (Fig. 2 (a)), under-actuated hands (Fig. 2 (b)), soft grippers (Fig. 2 (c)) and industrial systems (Fig. 3 (a-c)). 

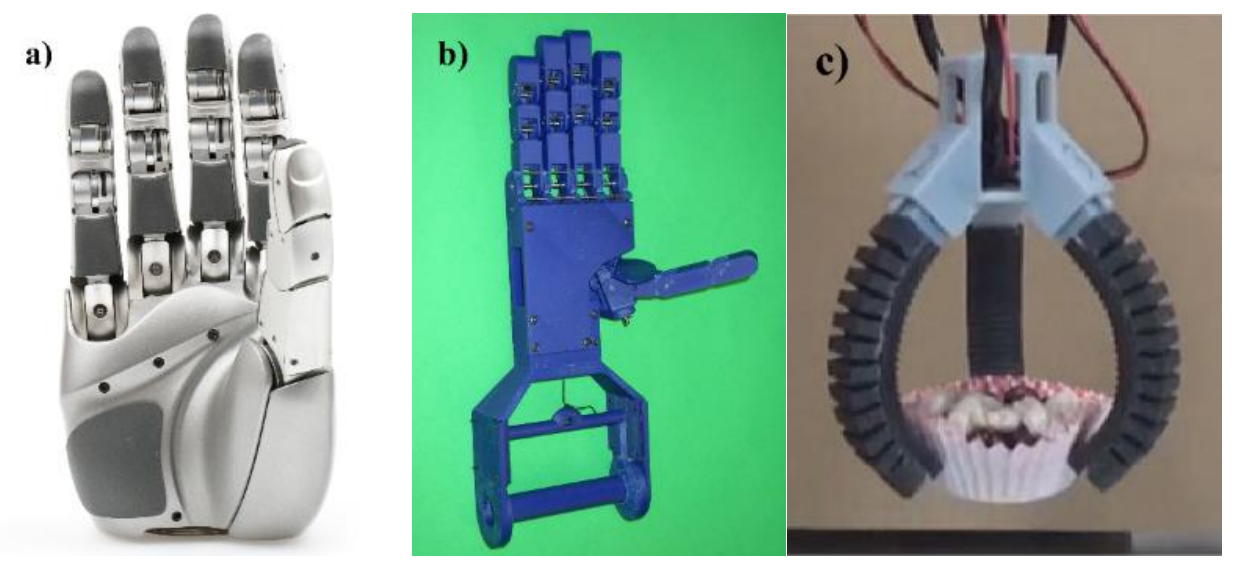

Fig. 2: (a) DLR hand HIT; (b) proposed under-actuated hand by Gosselin et al. (Gosselin et al., 2008); Wang and Hirai's soft gripper (Wang and Hirai, 2017).

\subsection{Anthropomorphic Hands}

A considerable part of research in robot end-effectors has targeted anthropomorphic grippers (hands), e.g. DLR (Butterfass et al., 2001) (see Fig. X (a)), Utah/MIT (Jacobsen et al., 1984), Stanford-JPL hand (Salisbury and Craig, 1982), Okada (Okada, 1982) hands and several others. These grippers aim to reach the dexterity of human hand by utilizing multi-degree-of-freedom (DOF) fingers, high resolution tactile sensor and vision systems (Kawasaki, 2015). The Shadow Dexterous Hand ("Shadow Dexterous Hand," 2017) with 24 joints with 17 DOFs and integrated position, tactile, force, temperature and pressure sensors is a commercial example of a versatile multi-finger robot gripper. While these grippers are capable of grasping objects of various shapes and complexity, they are confined to research laboratories due to their intricate control system, high price and high weight (Belter et al., 2013).

\subsection{Under-actuated Hands}

To overcome the control complexity of advanced multi-finger grippers, a wide range of under-actuated concepts have been proposed (Birglen et al., 2008). Dogan (Dogan, 2010) proposes a pneumatically actuated four-finger gripper that comprises 12 joints and 4 DOFs. As illustrated in Fig. X (b), Gosselin et al. (Gosselin et al., 2008) introduce a tendon-driven anthropomorphic under-actuated robotic gripper with 15 DOFs which operates with a single actuator. Even though under-actuated grippers are less complicated as regards mechanical components and control than advanced hands, their lifetime is significantly shorter than the industry standard (Belter et al., 2013).

\subsection{Soft Grippers}

Soft grippers (robotics) are another trendy MOGS that are evolved to balance out the simplicity and versatility of grippers. Over the last few years, several soft gripper concepts with different shapes and lengths are proposed (Connolly et al., 2017; Kim et al., 2013; Polygerinos et al., 2015; Shepherd et al., 2013; Wang and Hirai, 2017). Soft grippers are mostly designed as pneumatic networks (PneuNets), which consist of a series of usually 
square air segments (pockets) connected to one pressure source (Connolly et al., 2017). As fingers are actuated, pockets expand due to the air pressure, leading to deformation in the finger (Polygerinos et al., 2015). Wang and Hirai (Wang and Hirai, 2017) divide the finger into line-segments, where each segment has one air capsule; the segments are divided by sharp crevices on the backside of the finger, and strain-limiting material is on the front (inside) of the finger, as shown in Fig. X (c). When pressure is increased, the segments expand and push each other to cause bending. Shepherd et al. (Shepherd et al., 2013) use the principle of bellows, where large deformation is not necessary to expand the surface. The bellows make out the backside of the finger and strain-limiting material the inside, thus when air pressure is increased the bellows are extended. While soft grippers reduce the mechanical and algorithmic complexity involved in robot design, they suffer from low repeatability and accuracy (Tai et al., 2016).

\subsection{Industrial Systems}

Industrial MOGS, unlike the former general-purpose MOGS, are developed to execute ad hoc tasks. These MOGS are classified into custom-designed tool-change mechanisms, multi-gripper systems, and multi-function fingers (Causey, 1999).
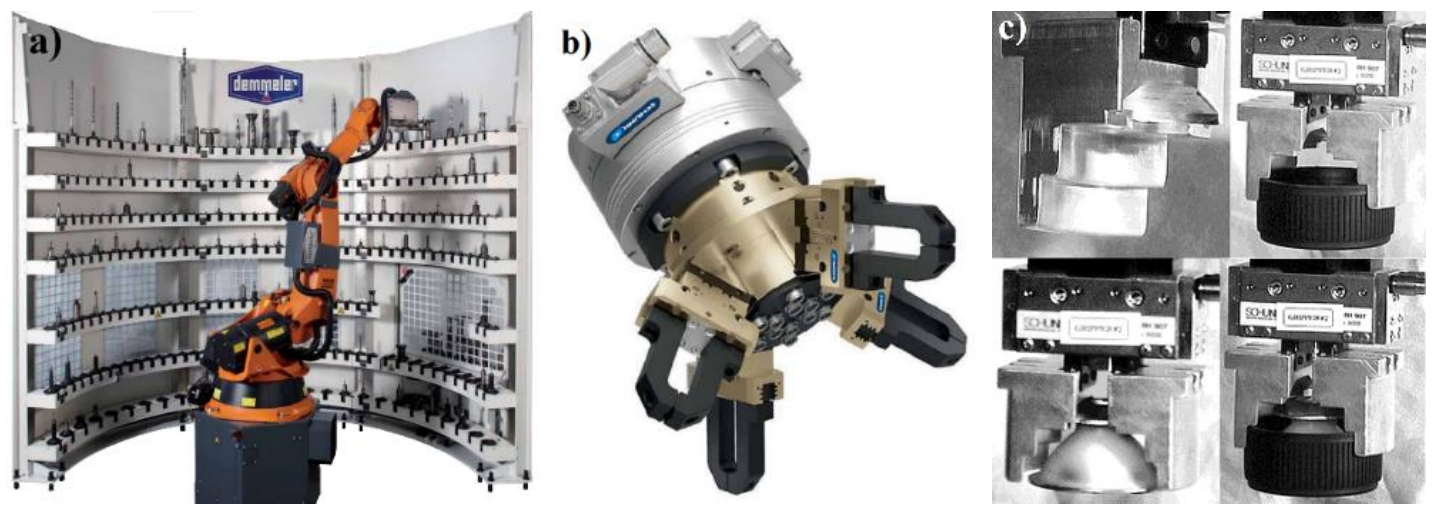

Fig. 3: Examples of: (a) tool change mechanisms ("Demmeler tool changer," 2017); (b) multi-gripper systems ("RVK-Schunk," 2017); multi-function fingers (Causey, 1999).

A tool-changer is a mechanism that is located in the robot station and allows the robot to change tools (gripper) in order to carry out different processes (Cotsman et al., 1991), as shown in Fig. 3 (a). These enable robots to access a broad range of tools and thus increases the workcell's productivity. However, tool-changing is a time-consuming operation and increases the station's cycle time and consequently reduces the cell's throughput.

Multi-gripper systems are rotary devices with 2-8 grippers that are fixed to the wrist of the robot and enable the robot to handle different workpieces by switching from one gripper to another (e.g. the RVK system from SCHUNK shown in Fig. 3 (b) ("RVK-Schunk," 2017)). Despite the fact that multi-gripper systems have minor impact on the cycle time and throughput of the robot cell in comparison to tool-changer mechanisms, they significantly reduce robots' total payload and functionality due to their heavy weight.

Multi-function fingers are designed to handle multiple specific workpieces without changing gripper or tools. As demonstrated in Fig. 3 (c), these MOGS are able to grasp multiple workpieces by using different sections of the fingers. Causey and Quinn (Causey 
and Quinn, 1998) point out that multi-function fingers are more effective, more productive, and cheaper than other industrial MOGSs (i.e. tool-changers and multi-gripper systems) as multi-function fingers do not add complexity to the control and structure of the robot and workcell. However, such fingers are manually designed today, which requires tremendous efforts and high skills and knowledge in robotics and mechanical designing. This solution is therefore generally used in applications for handling simple objects.

\section{Relevant Work}

Despite the fact that multi-function fingers are effective MOGS for increasing the throughput of a robot workcell, some limited research has been conducted on multifunction finger design automation and sadly none has proposed a universal solution. Here a brief overview is given, further outlined in (Honarpardaz et al., 2017b).

Pham et al. (Pham et al., 2005) propose a trial-and-error method which classifies workpieces into different groups based on their geometrical similarity (e.g. sphere, cube, cylinder, etc.). Their algorithm selects prescribed fingers (from a library) for one group of workpieces and then checks the quality of grasps when fingers grasp other groups of objects. If fingers cannot successfully grasp all groups, a new group of workpieces is selected to design fingers and the same process is continued to find a set of fingers that can grasp all workpieces. The proposed method is not able to guarantee a viable solution when workpieces have complex shapes and cannot fit into any of the predefined groups. Moreover, the success of the algorithm depends on the diversity of the workpieces' geometry. For instance, fingers that are designed for handling spherical objects cannot handle pyramidal ones and vice versa.

Velasco and Newman (Velasco and Newman, 1998) present a hypothesis to achieve multifunction fingers. The proposed CAD-based method begins with importing 3D models of all workpieces. This is followed by generating hypothetical geometries by superposing models of the workpieces. A set of solid blocks is then defined as initial fingers and the hypothetical superposed geometry is subtracted from the solid blocks. The remaining geometries of the initial fingers are considered to be multi-function fingers that can grasp all the workpieces. While this method is simple and quick to implement, it is exclusive to workpieces with different shapes and similar dimensions. As stated by the authors, the hypotheses cannot provide a feasible solution for cases where workpieces have similar geometry but different sizes (e.g. two balls with different radii).

\section{Proposed Method}

The generic optimized finger design method (GOFD) is categorized into four major process steps: geometry analysis, grasp planning and analysis, finger design, and experimental verifications. As the overview of the method (Fig. 4) demonstrates, the algorithm begins by analyzing the geometry of the workpieces and then planning and analyzing the grasp positions in order to handle the workpieces, i.e. how the workpieces should best be grasped. Once the grasps are known, the fingers are automatically designed and then manufactured, and finally they are experimentally verified. Each step consists of a set of sub-processes; these are explained in detail in the following sections. 


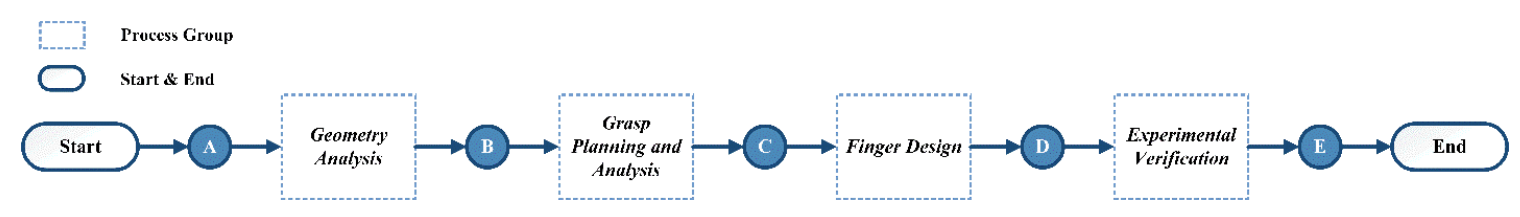

Fig. 4: Overview flowchart of the proposed GOFD method.

\subsection{Geometry Analysis}

Geometry analysis is the first step in the algorithm and carries out necessary inputs to the system. As illustrated in Fig. 5, the CAD model of the workpiece is first imported and the material properties of the fingers and the workpiece are defined. Following this step, specifications of the gripper (i.e. gripper mode, payload, maximum finger length, jaw stroke, maximum force) are provided to the algorithm (Honarpardaz et al., 2016a).

CAD models of workpieces with complex geometries usually contain several surfaces with various areas. In this work, a minimum surface area $\left(A_{\min }\right)$ is therefore defined (by the user) based on the type of gripper and the size of the fingers to discard ungraspable surfaces with small areas. In addition, a surface is then selected as a stable pose upon which the workpiece stands.

Once all the required inputs are known, two processes that run in parallel are initiated (see Fig. 5). The first parallel process begins by extracting coefficient of friction $(\mu)$ with respect to the fingers and workpiece materials from a defined library. Then, based on the coefficient of friction, the type of grasp (i.e. force or form closure), contact model (i.e. frictionless, hard finger and soft finger), and minimum number of required contact points (Nguyen, 1986) are determined (Honarpardaz et al., 2016a).

In the second parallel process, the workpiece is reoriented according to the defined stable pose. Later, every surface of the workpiece is recognized and its areas measured. Surfaces with areas $(A)$ smaller than $A_{\min }$ are disregarded and the remaining surfaces are then discretized and a point cloud model of the workpiece is created; otherwise, surfaces are considered to be ungraspable and discarded. This process reduces the size of point clouds and thereby the design process time (DPT). In the next step, surfaces' normal at each point of the workpiece is determined.



Fig. 5: Flowchart of geometry analysis group process in the GOFD method.

\subsection{Grasp Planning and Analysis}

The grasp planning and analysis step finds the grasp sets with the highest quality. In the proposed GOFD method, an optimization algorithm (in this case a Genetic Algorithm 
(GA)) is applied to the grasp planning process to reduce the computational burden of the process. A GA consists of a population of potential solutions where a set of individuals are evolved during a number of generations based on successive selection, crossover and mutation operations mimicking a Darwinian evolution process (Deb et al., 2002).

Fig. 6 presents the flowchart of the GA algorithm implemented in GOFD, inspired by (Daoud et al., 2011). The optimization starts with generating an initial population where each individual represents a grasp set with the minimum number of contact points $(n)$. Contact wrenches for each grasp set are then determined and convex hull of contact wrenches are calculated to obtain the wrench space $(w)$. The distance $(\varepsilon)$ between the origin of the wrench space $\left(w_{o}\right)$ and the closest hyperplane is measured to evaluate the quality of the grasp sets. The larger the $\varepsilon$, the higher the quality of the grasp (Ferrari and Canny, 1992). A new generation is then created based on the following rules:

- Successive selection, where the individuals (i.e. parents) with the highest fitness values are selected to create the next generation.

- Crossover, which combines the vector entries of a pair of parents to form children for the next generation.

- Mutation, where random changes are applied to the children in order to encourage broader exploration.

This process continues until the optimization criteria are satisfied or the number of generations reaches the maximum value. A set of grasp sets $(\psi)$ with the highest quality $\varepsilon$ is stored in a database to be used in the continuation of the proposed method.

In this optimization, the objective is to maximize grasp quality $(\varepsilon)$ while ensuring the grasp's stability (i.e. the origin of the wrench space $\left(w_{o}\right)$ lies inside the convex hull of the wrenches (Mishra et al., 1987; Salisbury, 1985)), as formulated in Eq. (1).

$$
\begin{aligned}
& \max _{\mathbf{x} \in \mathbb{N}} F(\mathbf{x}) \\
& g(\mathbf{x}) \leq 0 \\
& \left\{\begin{array}{l}
g(\mathbf{x})=0, \quad w_{o} \notin \text { ConvexHull }\left(\bigcup_{i=1}^{n}\left\{w_{i, 1}, \ldots, w_{i, 6}\right\}\right) \\
g(\mathbf{x})=1, \quad w_{o} \in \text { ConvexHull }\left(\bigcup_{i=1}^{n}\left\{w_{i, 1}, \ldots, w_{i, 6}\right\}\right)
\end{array}\right. \\
& 1 \leq x_{i} \leq m \\
& \mathbf{x}=\left[x_{1}, x_{2}, x_{3}, \ldots, x_{n}\right]
\end{aligned}
$$

where $x$ represents the integer variables (i.e. contact point IDs) and $m$ is the (known) size of the point cloud and the upper boundary of the variables. In a consequence, the optimization search space $(S)$ can be obtained by combination of $n$ from $m$ as shown in Eq. (2). 


$$
S=\left(\begin{array}{c}
m \\
n
\end{array}\right)=\frac{m !}{n !(m-n) !}
$$

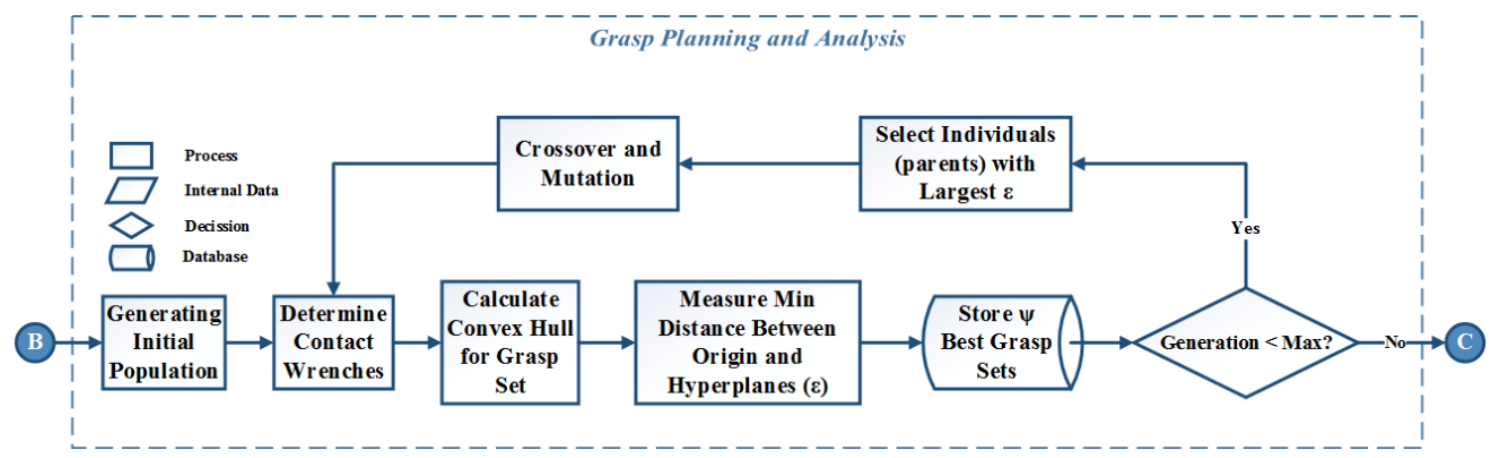

Fig. 6: Flowchart of grasp planning and analysis step of the GOFD method.

\subsection{Finger Design}

Once the group of best grasp sets $(\psi)$ has been obtained, the grasp set with the highest quality is selected to design the fingers. The first step in generating fingers is to design customized fingertips, as illustrated in Fig. 7. Fingertips are created based on pads with parametric dimensions that allow the user to modify the size of the fingertips based on the application after the actual fingers are generated. The pads are then projected onto the workpiece surface to imitate the surface contour of the workpiece at point contacts. Customizing fingertips increases the contact area and consequently a grasp's stability compared to conventional $\mathrm{V}$-groove and planar ones. In addition, the self-adjusting characteristic of customized fingertips compensates for the unavoidable position uncertainties in real applications and reduces failures (Honarpardaz et al., 2016a).

In cases where multi-objects are to be handled, the concavities of the contact areas for each workpiece are first measured and stored in a database. The process then returns to the beginning (point $\mathrm{A}$ ) and repeats the same procedure until the concavities of the contact areas of all workpieces have been measured and listed in the database. In the next step, fingertips for the first workpiece in the list (the workpiece with the lowest concavity) are created and considered to be the initial tips. The initial fingertips are then customized for the next workpiece in the list of workpieces. This procedure is repeated until the fingertips mimic all handling of workpieces (Honarpardaz et al., 2016b).

Following this step, parametric finger-bodies are generated. These are responsible for connecting the fingertips to the finger-base. Finger-bodies are designed according to the grasp type (i.e. internal or external), required offsets in different direction, and length and thickness of fingers. All these parameters will be accessible to the user after the finger design in case some modifications are needed.

In the next step, finger-bases are generated to connect the finger-bodies to the gripper jaws. Finger-bases are designed to adapt the interface of the previously defined gripper model. When all the components of the fingers (i.e. fingertips, finger-bodies and finger-bases) have been generated, the feasibility of the design is verified by checking for unwanted collisions between the workpieces and the fingers. Collision-free fingers are presented to the user to be manually inspected. If fingers are accepted by the user, CAD models of the 
fingers are exported for manufacturing; otherwise, the algorithm selects the next best grasp set from the grasp list and generates a new pair of fingers. This process continues until the user accepts the proposed design (Honarpardaz et al., 2016a). It should be noted that all steps from the user inspection to the end of the experimental process (point E) are executed manually.

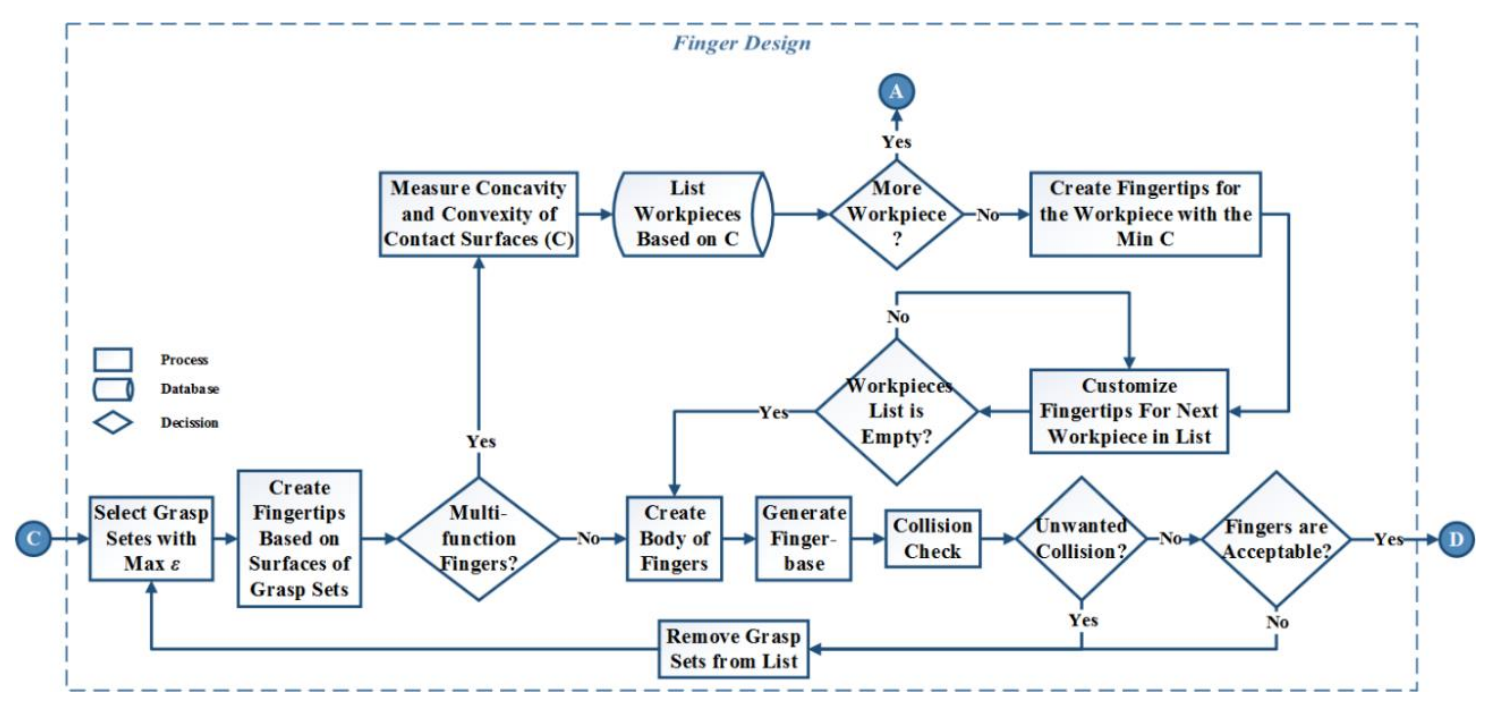

Fig. 7: Flowchart of the finger design group process in the GOFD method.

\subsection{Experimental Verification}

Experimental verification is the most important step to obtain final verification and validation of any finger design process. However, it is uncommon due to the cost of the required hardware and a lack of necessary know-how in setting up the experiment. In (Honarpardaz et al., 2017a), a generic experimental method to verify robot fingers is proposed, consisting of grasp stability and performance verification of fingers. As presented in Fig 8, GOFD uses the same approach to verify the designed fingers. This step begins with manufacturing the fingers and mounting them on the robot manipulator. In order to verify the stability of the fingers, force and torque experiments are conducted to determine the maximum disturbance forces and torques that fingers can resist in the $X, Y$ and $Z$ directions. If the stability of the fingers satisfies the requirements, the performance of fingers is examined; otherwise, a new grasp set is selected and the same process is followed. Performance verification contains two steps: pick-and-place and assembly experiments. In pick-and-place experiment, fingers are examined by picking workpieces and placing them in a predefined position. The assembly experiment measures the performance of fingers in an assembly task. Contingent upon the robot application, one or both of the performance experiments may be conducted. Finally, the design process terminates once performance experiments have been executed and fingers satisfy the expected performance. The reader is referred to (Honarpardaz et al., 2017a) for details of grasp stability and performance experiments. 


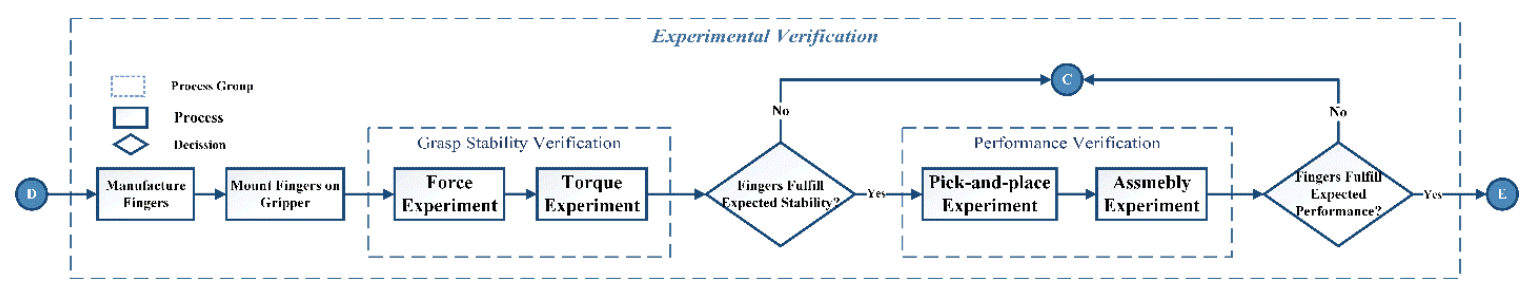

Fig. 8: Flowchart of the experimental verification group process in the GOFD method.

\section{Case Study}

The proposed GOFD method is implemented in VBA as the application programming interface (API) of CATIA. The geometry analysis and finger design processes are carried out in CATIA V5 and the grasp planning and analysis process is solved in MATLAB. The genetic algorithm (GA) toolbox implemented in MATLAB is utilized to find the optimum grasp set. In the GA, population size and maximum number of generations are set to 500 and 150, respectively. As stated previously, optimization variables are integer-valued and their number is equivalent to the number of contact points (i.e. two in theses case studies). In order to verify the functionality of the proposed method, 24 common commercial components from actual robot applications in various industries were selected as case studies. As shown in Table 1, the selected components vary in shape and complexity in order to truly examine the general applicability and functionality of the method. Furthermore, selected components are paired to generate multi-function fingers. 
Table 1: Selected pairs of industrial components for multi-function finger design case studies.

\begin{tabular}{|c|c|c|c|c|c|c|c|c|c|c|c|}
\hline $\begin{array}{l}\text { Pair } \\
\text { No. }\end{array}$ & $\begin{array}{c}\text { Workpiece } \\
\text { Name }\end{array}$ & $\begin{array}{c}\text { Workpiece } \\
\text { CAD Model }\end{array}$ & $\begin{array}{l}\text { Pair } \\
\text { No. }\end{array}$ & $\begin{array}{c}\text { Workpiece } \\
\text { Name }\end{array}$ & $\begin{array}{c}\text { Workpiece } \\
\text { CAD Model }\end{array}$ & $\begin{array}{l}\text { Pair } \\
\text { No. }\end{array}$ & $\begin{array}{c}\text { Workpiece } \\
\text { Name }\end{array}$ & $\begin{array}{c}\text { Workpiece } \\
\text { CAD Model }\end{array}$ & $\begin{array}{l}\text { Pair } \\
\text { No. }\end{array}$ & $\begin{array}{c}\text { Workpiece } \\
\text { Name }\end{array}$ & $\begin{array}{c}\text { Workpiece } \\
\text { CAD Model }\end{array}$ \\
\hline \multirow{2}{*}{1} & $\begin{array}{l}\text { Phone } \\
\text { PCB }\end{array}$ & & \multirow{2}{*}{4} & $\begin{array}{c}\text { AAA } \\
\text { Battery }\end{array}$ & & \multirow{2}{*}{7} & Hexnut & & \multirow{2}{*}{10} & $\begin{array}{c}\text { Nozzle } \\
\text { Spanner }\end{array}$ & \\
\hline & $\begin{array}{l}\text { Phone } \\
\text { Cover }\end{array}$ & & & Gasket & & & Bolt & & & Servo & \\
\hline \multirow{2}{*}{2} & Clip & & \multirow{2}{*}{5} & Slot & & \multirow{2}{*}{8} & $\begin{array}{l}\text { Gripper } \\
\text { Platform }\end{array}$ & & \multirow{2}{*}{11} & Conflat & \\
\hline & Board & & & Cylinder & & & $\begin{array}{c}\text { Minifit } \\
600\end{array}$ & & & USB & \\
\hline \multirow{2}{*}{3} & $\begin{array}{c}\text { Lamp } \\
\text { Base }\end{array}$ & & \multirow{2}{*}{6} & $\begin{array}{c}\text { DSUB } \\
\text { Female }\end{array}$ & & \multirow{2}{*}{9} & $\begin{array}{l}\text { Minifit } \\
\text { Plug }\end{array}$ & & \multirow{2}{*}{12} & Concave & \\
\hline & Lamp Cap & & & $\begin{array}{l}\text { DSUB- } \\
\text { Male }\end{array}$ & & & $\begin{array}{c}\text { Minifit } \\
\mathbf{2 0 0}\end{array}$ & & & Box & \\
\hline
\end{tabular}

\section{Result}

This section presents the results of applying the GOFD method on the components defined in Table 1. It consists of two sub-sections: finger design, which illustrates the resulting fingers designed by GOFD, and experimental verification, where the performance of the designed fingers is evaluated.

\subsection{Finger Design}

Design process time (DPT) and grasp quality $(\varepsilon)$ using GAFD and GOFD methods for the selected case studies are illustrated in Table 2. The DPT ratio of GAFD to GOFD is also shown to highlight the efficiency of the optimization algorithm. Further, in order to properly compare the quality of grasps synthesized with GAFD and GOFD, the grasp quality is normalized based on the absolute best grasp obtained from the GAFD method and the difference in percentage is also presented in the table. Note that GAFD includes an exhaustive search and hence the best theoretical grasp is always identified, as compared to GOFD that uses a heuristic search method, the GA, to search for an optimal grasp. For example, the first row in Table 2 shows that the design time is reduced 200 times using the proposed method, with a quality loss of only $0.34 \%$. For all case studies, the proposed method is able to reduce the design time by an average of 16600 seconds. The grasp quality loss from using GOFD is also low, with an average of $0.47 \%$ and the highest loss being just $2.1 \%$. 
Table 2: Design process time (DPT) and grasp quality $(\varepsilon)$ of case studies using the GAFD and GOFD methods.

\begin{tabular}{|c|c|c|c|c|c|c|c|}
\hline Workpiece Name & $\begin{array}{c}\text { Size of Point } \\
\text { Cloud }\end{array}$ & $\begin{array}{c}D P T_{G A F D} \\
{[s]}\end{array}$ & $\begin{array}{c}D P T_{G O F D} \\
{[s]}\end{array}$ & $\overline{D P T}$ & $\begin{array}{c}\text { Normalized } \\
\varepsilon_{G A F D}\end{array}$ & $\begin{array}{c}\text { Normalized } \\
\varepsilon_{G O F D}\end{array}$ & $\varepsilon_{\text {dif }}[\%]$ \\
\hline Phone PCB & 5532 & 45702 & 180 & 253.5 & 11.26 & 11.23 & 0.34 \\
\hline Phone Cover & 8512 & 161467 & 312 & 517.7 & 17.82 & 17.52 & 1.71 \\
\hline Clip & 1181 & 1300 & 155 & 8.4 & 12.13 & 12.07 & 0.53 \\
\hline Board & 6891 & 83119 & 235 & 353.8 & 9.72 & 9.62 & 1.05 \\
\hline Lamp Base & 3577 & 18030 & 176 & 102.3 & 5.00 & 4.99 & 0.26 \\
\hline Lamp Cap & 1520 & 2831 & 173 & 16.4 & 10.28 & 10.15 & 1.19 \\
\hline Battery & 1088 & 1125 & 157 & 7.2 & 2.06 & 2.06 & 0 \\
\hline Gasket & 544 & 255 & 144 & 1.8 & 3.19 & 3.19 & 0 \\
\hline Slot & 776 & 472 & 77 & 6.2 & 3.76 & 3.73 & 0.72 \\
\hline Cylinder & 2692 & 8897 & 199 & 44.6 & 6.61 & 6.60 & 0.13 \\
\hline DSUB Female & 3944 & 20040 & 206 & 97.2 & 3.72 & 3.70 & 0.50 \\
\hline DSUB Male & 2948 & 10010 & 187 & 53.5 & 2.41 & 2.41 & 0.21 \\
\hline Hexnut & 2098 & 5616 & 190 & 29.5 & 3.29 & 3.29 & 0 \\
\hline Bolt & 2794 & 10100 & 207 & 48.8 & 9.79 & 9.58 & 2.16 \\
\hline Gripper Platform & 2008 & 3998 & 164 & 24.3 & 5.05 & 5.05 & 0 \\
\hline Minifit 600 & 2696 & 8770 & 179 & 49 & 2.75 & 2.75 & 0.01 \\
\hline Minifit Plug & 1168 & 1362 & 158 & 8.6 & 4.34 & 4.31 & 0.55 \\
\hline Minifit 200 & 698 & 428 & 148 & 2.9 & 3.07 & 3.06 & 0.16 \\
\hline Nozzle Spanner & 2713 & 9599 & 203 & 47.2 & 9.62 & 9.50 & 1.19 \\
\hline Servo & 1175 & 1386 & 164 & 8.5 & 5.37 & 5.36 & 0.08 \\
\hline Conflat & 1681 & 2907 & 170 & 17.1 & 5.55 & 5.51 & 0.70 \\
\hline USB & 893 & 787 & 163 & 4.8 & 5.01 & 5.01 & 0 \\
\hline Concave & 358 & 134 & 101 & 1.3 & 3.06 & 3.06 & 0 \\
\hline Box & 2166 & 4223 & 100 & 42.4 & 5.25 & 5.25 & 0 \\
\hline Average & 2485.5 & 16773.3 & 172.8 & 72.2 & 6.25 & 6.21 & 0.47 \\
\hline
\end{tabular}

The results from the designed multi-function fingers for each pair of workpieces are shown in Table 3, wherein columns represent 3D models of the left and right fingers and fingertips in each case. As shown in the table, the fingertips are customized to be able to imitate the contact surfaces of both workpieces in each of the different cases. 
Table 3: CAD model of designed multi-function fingers and fingertips in case studies using GOFD method.

\begin{tabular}{|c|c|c|c|c|c|c|c|c|c|c|c|}
\hline $\begin{array}{l}\text { Pair } \\
\text { No. }\end{array}$ & $\begin{array}{c}\text { Workpiece } \\
\text { Name }\end{array}$ & $\begin{array}{c}\text { Left } \\
\text { Finger }\end{array}$ & $\begin{array}{c}\text { Right } \\
\text { Finger }\end{array}$ & $\begin{array}{c}\text { Left } \\
\text { Fingertip }\end{array}$ & $\begin{array}{c}\text { Right } \\
\text { Fingertip }\end{array}$ & $\begin{array}{l}\text { Pair } \\
\text { No. }\end{array}$ & $\begin{array}{c}\text { Workpiece } \\
\text { Name }\end{array}$ & $\begin{array}{c}\text { Leff } \\
\text { Finger }\end{array}$ & $\begin{array}{c}\text { Right } \\
\text { Finger }\end{array}$ & $\begin{array}{c}\text { Left } \\
\text { Fingertip }\end{array}$ & $\begin{array}{c}\text { Right } \\
\text { Fingertip }\end{array}$ \\
\hline \multirow{2}{*}{1} & $\begin{array}{c}\begin{array}{c}\text { Phone } \\
\text { PCB }\end{array}\end{array}$ & & & & & & Hexnut & & & & \\
\hline & $\begin{array}{l}\text { Phone } \\
\text { Cover }\end{array}$ & & & & & & Bolt & & & & \\
\hline \multirow{2}{*}{2} & Clip & & & & & & $\begin{array}{l}\text { Gripper } \\
\text { Platform }\end{array}$ & & & & \\
\hline & Board & & & & &  & $\begin{array}{c}\text { Minifit } \\
600\end{array}$ & & & & \\
\hline \multirow{2}{*}{3} & $\begin{array}{l}\text { Lamp } \\
\text { Base }\end{array}$ & & & & & & $\begin{array}{l}\text { Minifit } \\
\text { Plug }\end{array}$ & & & & \\
\hline & $\begin{array}{c}\text { Lamp } \\
\text { Cap }\end{array}$ & & & & & & $\begin{array}{c}\text { Minifit } \\
200\end{array}$ & & & & \\
\hline \multirow{2}{*}{4} & $\begin{array}{c}\text { AAA } \\
\text { Battery }\end{array}$ & & & & & & $\begin{array}{l}\text { Nozzle } \\
\text { Spanner }\end{array}$ & & & & \\
\hline & Gasket & & & & & & Servo & & & & \\
\hline \multirow{2}{*}{5} & Slot & & & & & & Conflat & & & & \\
\hline & Cylinder & & & & & & USB & & & & \\
\hline \multirow{2}{*}{6} & $\begin{array}{c}\text { DSUB } \\
\text { Female }\end{array}$ & & & & & & Concave & & & & \\
\hline & $\begin{array}{c}\text { DSUB } \\
\text { Male }\end{array}$ & & & & & & Box & & & & \\
\hline
\end{tabular}

\subsection{Experimental Verification}

In order to validate the functionality of the fingers they are manufactured and an experimental verification is conducted. In this study, additive manufacturing is used to produce prototypes of fingers in acrylonitrile butadiene styrene (ABS) plastic using a Stratasys U-Print SE Plus 3D printer. An IRB 14000 (YuMi) robot from ABB is used as the platform to perform the experiments. In order to examine grasp stability and performance of the fingers, the same experimental method and setup suggested by Honarpardaz et al. (Honarpardaz et al., 2017a) are employed. As shown in Fig. 9, three pairs (pair 5, 7 and 12) were selected for grasp stability and performance verification. 


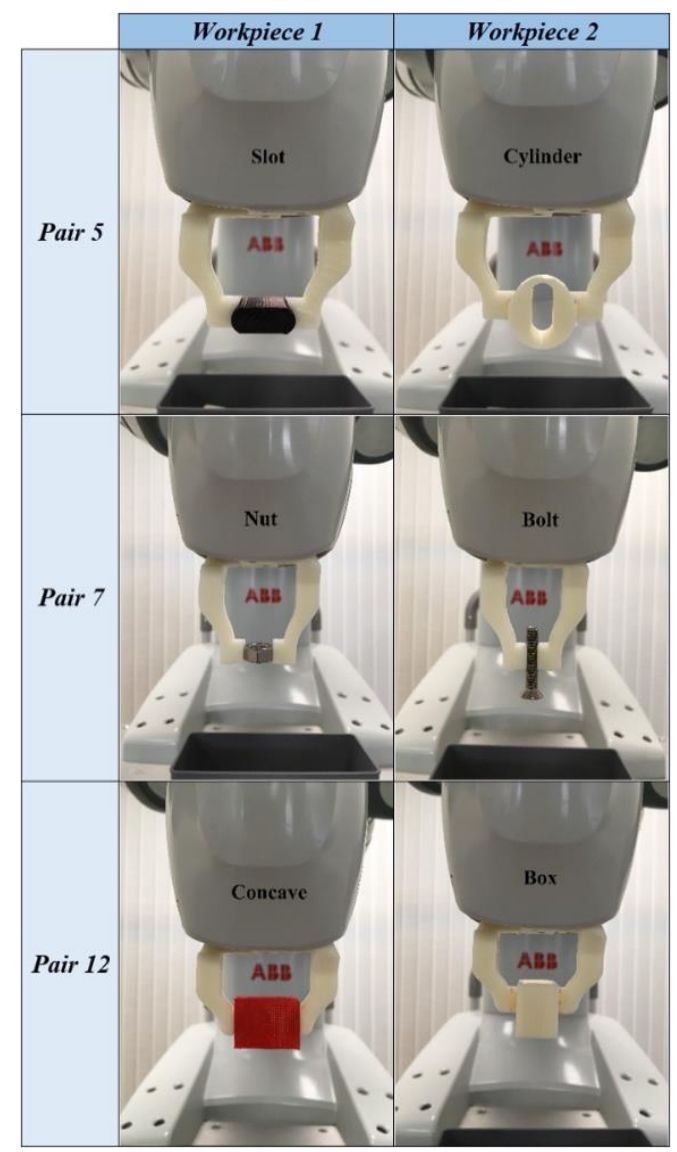

Fig. 9: Prototype of selected case studied for experimental verifications.

\subsubsection{Grasp Stability Verification}

Results of grasp stability, i.e. force and torque experiments, are displayed in Figs. 10 and 11. In Fig. 10, stability (resistance) of single-function fingers is compared with multifunction ones when external forces in different directions are exerted on the workpieces. Stability of the single-function and multi-function fingers against external torques are presented in Fig. 11. It should be noted that grasp stability experiments are executed only in directions perpendicular to the force closure (Nguyen, 1986) grasps. To facilitate the comparison of stability of fingers, form closure grasp (Bicchi, 1995) directions are represented by the maximum holding force (i.e. $20[\mathrm{~N}]$ ) and torque (i.e. $0.5[\mathrm{~N} . \mathrm{m}]$ ) of the robot manipulator without damaging the hardware. Form closure grasps are considered to be the most robust type of grasps; the more form closure grasp directions, the more reliable fingers. In both force and torque experiments, five iterations are conducted for each experiment in order to reduce possible external errors. Standard deviation, which is low, with an average of $\pm 4 \%$, is shown for all experiments.

According to the results, both single- and multi-function fingers produce form closure grasps in the same directions. However, the resistibility of single-function fingers against disturbance loads is greater than that of multi-function fingers. This is due to the fact that the multi-function fingers have smaller contact areas for each workpiece than singe function fingers. 


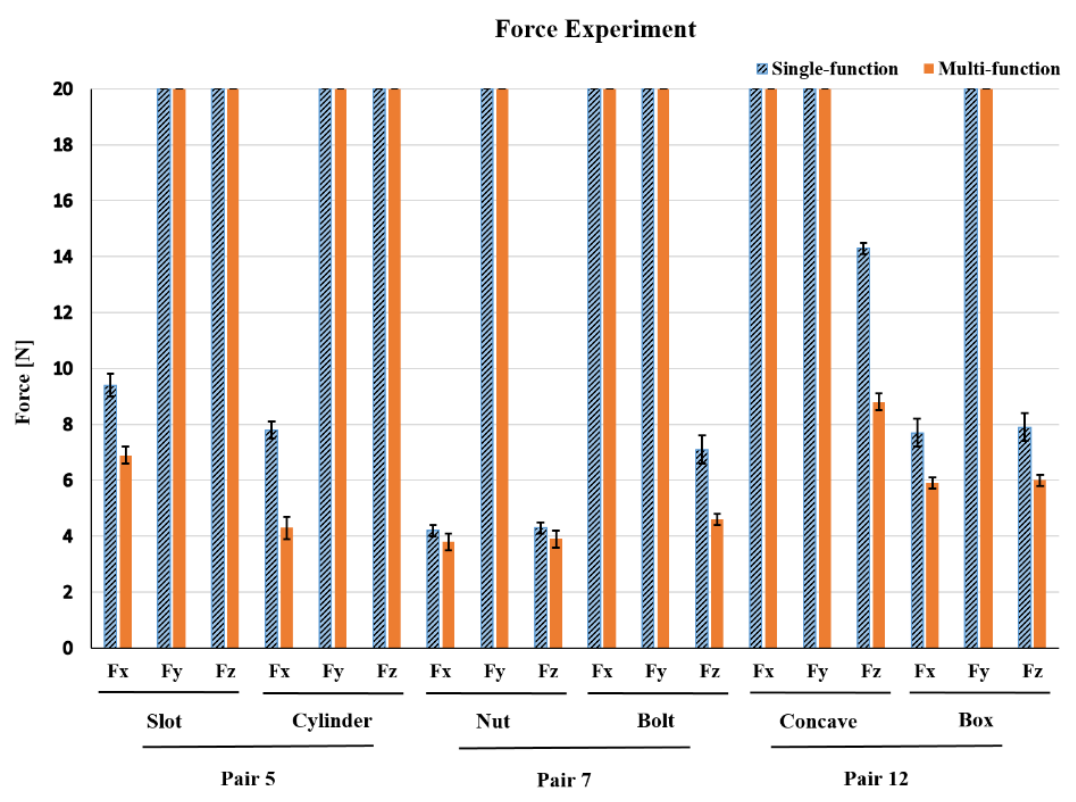

Fig. 10. Results of the force experiment in the $\mathrm{X}, \mathrm{Y}$ and $\mathrm{Z}$ direction.

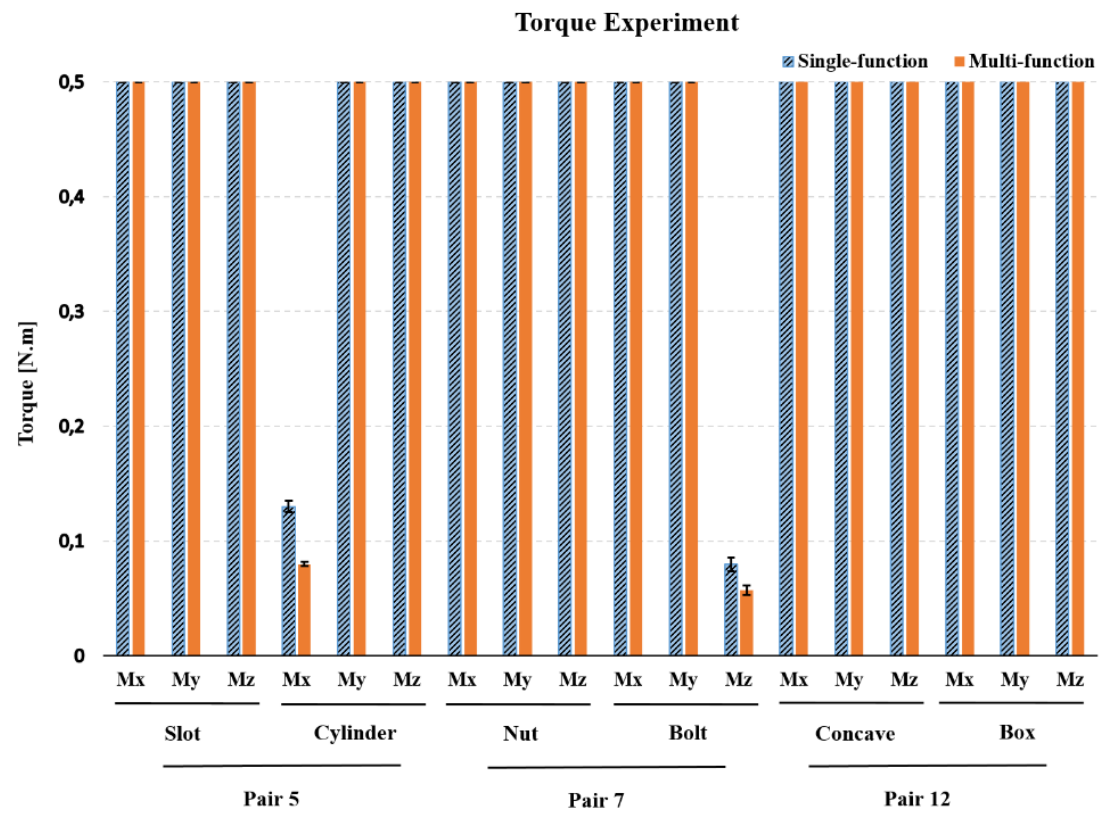

Fig. 11. Results of the torque experiment in the $\mathrm{X}, \mathrm{Y}$ and $\mathrm{Z}$ direction.

\subsubsection{Performance Verification}

To measure the performance and repeatability of the fingers, a pick-and-place task is set up as shown in Fig. 12. In this experiment (see Extension 1), workpieces are picked from feeders with a certain position and location and placed in a predefined container. The same series of experiments are conducted using single-function and multi-function fingers to be able to properly compare the performance of the fingers. For each case study, 100 iterations were executed and both single- and multi-function fingers had $100 \%$ successful operations. 




Fig. 12: Performance experimental verification setup.

Extension 1: Experimental verification of GOFD multi-functional fingers.

\section{Discussion}

This section analyzes and benchmarks the results of the existing generic automated finger design (GAFD) method against the optimized version of (GOFD). Design process time, grasp quality, grasp stability, and the performance of the fingers are comprehensively discussed in the following subsections.

\subsection{Design Process Time}

As can be seen from Fig. 13 (a, b), utilizing the genetic algorithm in the grasp planner module significantly reduces the design process time. In the extreme case of the phone cover with an 8512-point cloud model, the DPT using GOFD is more than 500 times faster than GAFD (see Table 2), which is equivalent to a saving of 44.8 hours. Fig. 13 (b) also indicates that the complexity and point cloud size of workpieces have an insignificant impact on DPT using the genetic algorithm in GOFD compared to the exhaustive search used in GAFD. Table 2 implies that the performance of the GOFD method is more perceptible as implemented on workpieces with intricate geometries. 
a)

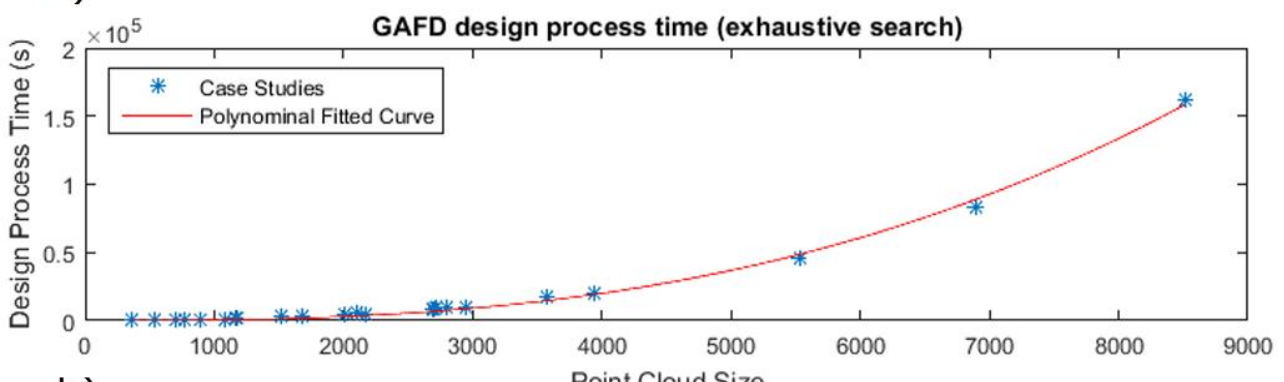

b)

Point Cloud Size

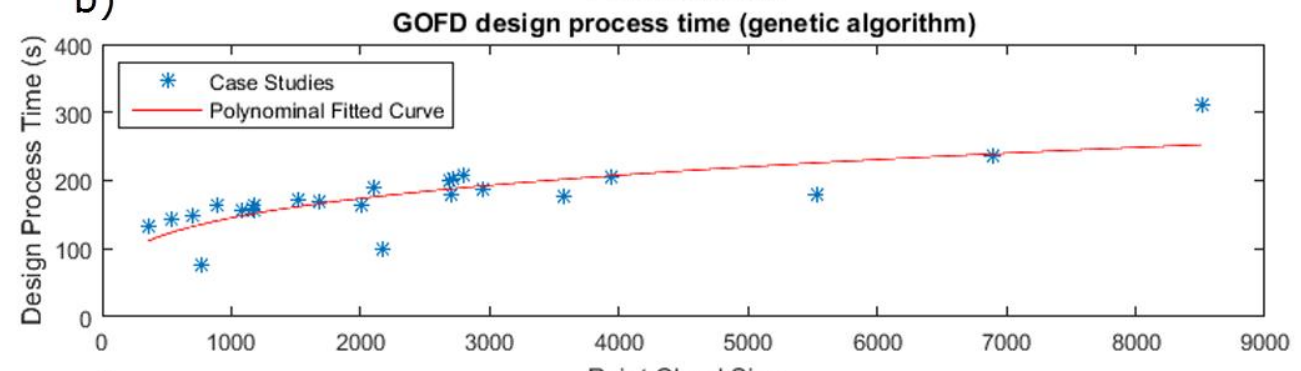

c)

Point Cloud Size

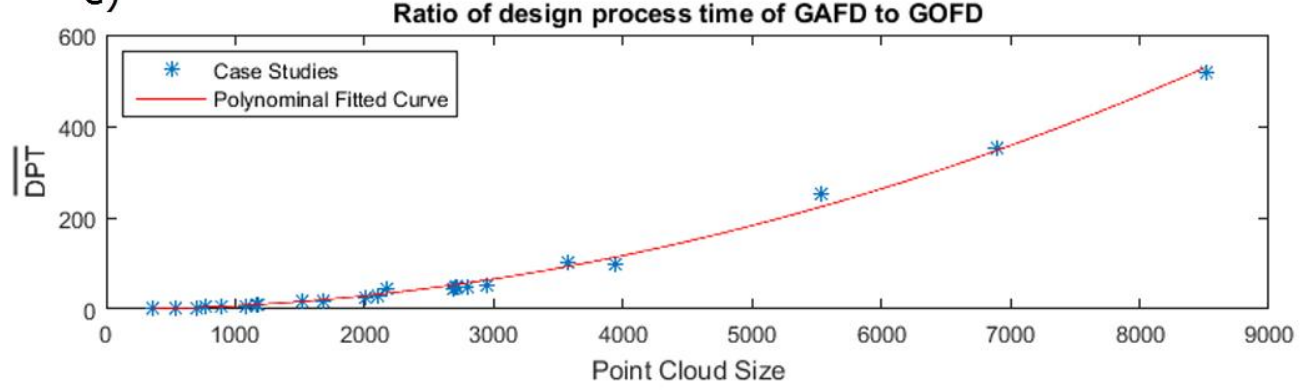

Fig. 13: Design process time of (a) the GAFD and (b) the GOFD methods, and (c) ratio of the design process time of GAFD to GOFD.

\subsection{Grasp Quality}

While the design process time of fingers is substantially reduced in GOFD, the quality of grasps is comparable to GAFD, as expressed in Table 2. In the worst case study (Lamp Base), grasp quality has $2.16 \%$ deviation from the absolute best grasp known from the exhaustive search method. However, the degradation in grasp quality using GOFD is less than $1 \%$ in $80 \%$ of the fingers. Furthermore, as shown in Fig. 14, the point cloud size and the grasp quality have low correlation (a coefficient of $\rho=0.47$ ). The complexity of the geometry and the randomly selected initial population of the GA might however have some influences on the results. 


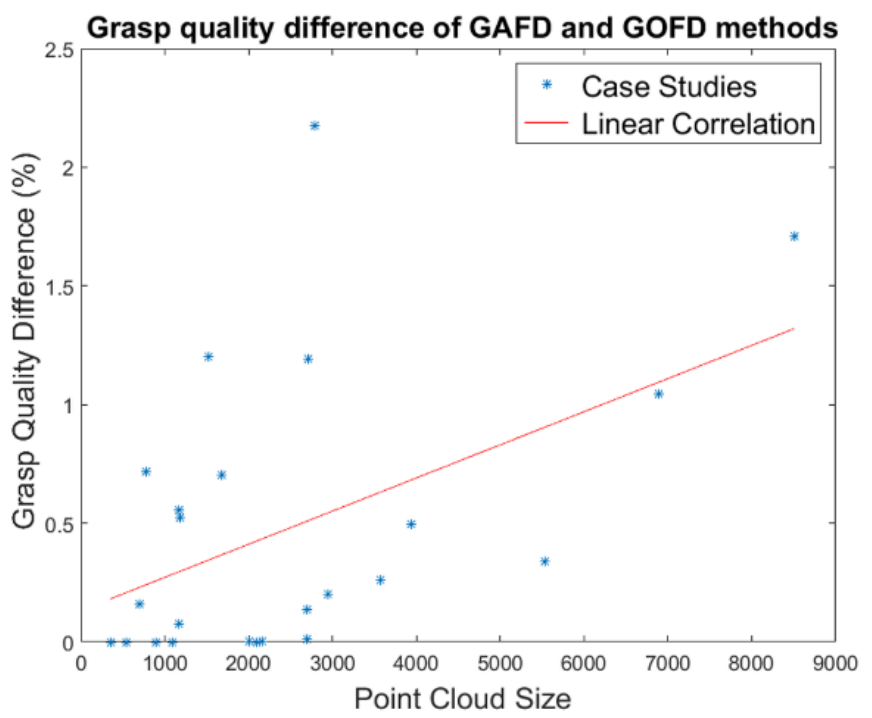

Fig. 14: Grasp quality difference of the GOFD and GAFD method vs. point cloud size of workpieces.

\subsection{Grasp Stability}

As results presented in Fig. 10 and 11 show, customized fingertips impose a form closure grasp of workpieces in at least four directions. As a consequence, the stability of the fingers is increased as they are less dependent on the friction force between the fingers and workpiece resulting from the applied grasping force. However, comparing grasp stability of multi-function fingers with single-function ones reveals that multi-function fingers on average have $28 \%$ lower grasp stability in force closure directions. This is due to the multifunction fingers dedicating $50 \%$ of the fingertip surface area for each workpiece, where a smaller contact area leads to lower resistance to external loads (i.e. force and torque) compared to single-function fingers.

\subsection{Performance}

Despite the fact that grasp stability of multi-function fingers is relatively less than singlefunction fingers, results of performance verification indicate that both single- and multifunction fingers have the same $(100 \%)$ success rate in prescribed pick-and-place tasks. In pick-and-place applications, however, disturbance loads are limited to gravity and inertia which are inconsequential in these case studies due to the small masses of the workpieces. Lower performance of multi-function fingers might therefore be attributed to smaller contact areas in cases where significant disturbance loads are exerted on workpieces.

\section{Conclusion}

This work presents a generic optimized method to automate the design process of fingers for industrial robot grippers. Despite the high demands of robot industries, there is a lack

of formal approaches to automate the design of reliable multi-function fingers. The generic 
optimized finger design method (GOFD) has been developed to enable a non-expert user to design functional fingers within a short time frame. The proposed method is applied to 24 industrial components and the designed fingers are experimentally verified. The average design process time in GOFD for the defined case studies is 148 seconds and the average grasp quality has $0.47 \%$ deviation from the absolute best grasp. Reducing $98.6 \%$ of the computational time with a quality loss of just $0.47 \%$ is a promising result for the proposed method. Furthermore, the GOFD method could handle both single and multi-function fingers, as well as force and form closure grasp, regardless of the geometrical complexity of the workpieces. Although the proposed method allows internal and external grasp approaches for single-function fingers' design, both grasp approaches cannot concurrently be considered for multi-function fingers' design. The introduced GOFD method can help robot industries comply with the trending agile market. Scholars who are inexpert in robotics may also benefit from utilizing GOFD in their research to generate functional fingers.

\section{Acknowledgements}

The research leading to these results has received funding from the European Community's Framework Programme Horizon 2020 - under Grant Agreement No. 644938 - SARAFun. Xiaolong Feng and Jonas Larsson at ABB are gratefully acknowledged for technical discussions and various support. Special thanks go to Karthik Sathyanarayana for implementing the algorithm on different objects to verify the method.

\section{Reference}

Belter, J.T., Segil, J.L., Dollar, A.M., Weir, R.F., 2013. Mechanical design and performance specifications of anthropomorphic prosthetic hands: a review. J. Rehabil. Res. Dev. 50, 599-618.

Bicchi, A., 1995. On the Closure Properties of Robotic Grasping. Int. J. Robot. Res. 14, $319-334$. doi:10.1177/027836499501400402

Birglen, L., Lalibert, T., Gosselin, C.M., 2008. Underactuated Robotic Hands, 1st ed. Springer Publishing Company, Incorporated.

Butterfass, J., Grebenstein, M., Liu, H., Hirzinger, G., 2001. DLR-Hand II: next generation of a dextrous robot hand, in: Proceedings 2001 ICRA. IEEE International Conference on Robotics and Automation (Cat. No.01CH37164). Presented at the Proceedings 2001 ICRA. IEEE International Conference on Robotics and Automation (Cat. No.01CH37164), pp. 109-114 vol.1. doi:10.1109/ROBOT.2001.932538

Causey, G.C., 1999. Elements of agility in manufacturing. PhD Diss. Dep. Mech. Aerosp. Eng. Case West. Reserve Univ.

Causey, G.C., Quinn, R.D., 1998. Gripper design guidelines for modular manufacturing, in: 1998 IEEE International Conference on Robotics and Automation, 1998. Proceedings. Presented at the 1998 IEEE International Conference on Robotics and Automation, 1998. Proceedings, pp. 1453-1458 vol.2. doi:10.1109/ROBOT.1998.677309

Connolly, F., Walsh, C.J., Bertoldi, K., 2017. Automatic design of fiber-reinforced soft actuators for trajectory matching. Proc. Natl. Acad. Sci. 114, 51-56. doi:10.1073/pnas.1615140114

Cotsman, D.N., Kovach, J., Zuccaro, D.C., Kudwa, F.J., 1991. Robot tool changer and overload device. US4995493 A.

Daoud, N., Gazeau, J.P., Zeghloul, S., Arsicault, M., 2011. A fast grasp synthesis method for online manipulation. Robot. Auton. Syst. 59, 421-427. doi:10.1016/j.robot.2011.02.005

Deb, K., Pratap, A., Agarwal, S., Meyarivan, T., 2002. A fast and elitist multiobjective genetic algorithm: NSGA-II. IEEE Trans. Evol. Comput. 6, 182-197. doi:10.1109/4235.996017

Demmeler tool changer [WWW Document], 2017. URL https://www.demmeler.com/products-shop/automationtechnology/tool-arena/ (accessed 7.12.17).

Dogan, B., 2010. Development of a two-fingered and a four-fingered robotic gripper. Middle East Technical University, The Graduate School Of Natural And Applied Science.

Factory-in-a-day [WWW Document], 2017. URL http://www.factory-in-a-day.eu/ (accessed 6.12.16). 
Ferrari, C., Canny, J., 1992. Planning optimal grasps, in: , 1992 IEEE International Conference on Robotics and Automation, 1992. Proceedings. Presented at the , 1992 IEEE International Conference on Robotics and Automation, 1992. Proceedings, pp. 2290-2295 vol.3. doi:10.1109/ROBOT.1992.219918

Gosselin, C., Pelletier, F., Laliberte, T., 2008. An anthropomorphic underactuated robotic hand with 15 dofs and a single actuator, in: 2008 IEEE International Conference on Robotics and Automation. Presented at the 2008 IEEE International Conference on Robotics and Automation, pp. 749-754. doi:10.1109/ROBOT.2008.4543295

Greg Causey, 2003. Guidelines for the design of robotic gripping systems. Assem. Autom. 23, 18-28. doi:10.1108/01445150310460033

Honarpardaz, M., Tarkian, M., Feng, X., Sirkett, D., Ölvander, J., 2016a. Generic Automated Finger Design. p. V05BT07A071. doi:10.1115/DETC2016-60514

Honarpardaz, M., Tarkian, M., Ölvander, J., Feng, X., 2017a. Experimental verification of design automation methods for robotic finger. Robot. Auton. Syst. 94, 89-101. doi:10.1016/j.robot.2017.04.011

Honarpardaz, M., Tarkian, M., Ölvander, J., Feng, X., 2017b. Finger design automation for industrial robot grippers: A review. Robot. Auton. Syst. 87, 104-119. doi:10.1016/j.robot.2016.10.003

Honarpardaz, M., Tarkian, M., Sirkett, D., Ölvander, J., Feng, X., Elf, J., Sjögren, R., 2016b. Generic Automated Multifunction Finger Design. IOP Conf. Ser. Mater. Sci. Eng. 157, 012015. doi:10.1088/1757-899X/157/1/012015

Jacobsen, S.C., Wood, J.E., Knutti, D.F., Biggers, K.B., 1984. The UTAH/M.I.T. Dextrous Hand: Work in Progress. Int. J. Robot. Res. 3, 21-50. doi:10.1177/027836498400300402

Kawasaki, H., 2015. Robot Hands and Multi-Fingered Haptic Interfaces: Fundamentals and Applications. World Scientific Publishing Co, New Jersey.

Kim, S., Laschi, C., Trimmer, B., 2013. Soft robotics: a bioinspired evolution in robotics. Trends Biotechnol. 31, 287294. doi:10.1016/j.tibtech.2013.03.002

Mishra, B., Schwartz, J.T., Sharir, M., 1987. On the existence and synthesis of multifinger positive grips. Algorithmica 2, 541-558. doi:10.1007/BF01840373

Nguyen, V.-D., 1986. Constructing force-closure grasps, in: 1986 IEEE International Conference on Robotics and Automation. Proceedings. Presented at the 1986 IEEE International Conference on Robotics and Automation. Proceedings, pp. 1368-1373. doi:10.1109/ROBOT.1986.1087483

Nof, S.Y. (Ed.), 1999. Handbook of Industrial Robotics, 2 edition. ed. Wiley, New York.

Okada, T., 1982. Computer Control of Multijointed Finger System for Precise Object-Handling. IEEE Trans. Syst. Man Cybern. 12, 289-299. doi:10.1109/TSMC.1982.4308818

Pham, D.T., Gourashi, N.S., Eldukhri, E.E., 2005. Intelligent conceptual design of robot grippers for assembly tasks, in: Pham, D.T., Eldukhri, E.E., Soroka, A.J. (Eds.), Intelligent Production Machines and Systems: First I*PROMS Virtual Conference, 4-15 July 2005. Presented at the Virtual International Conference on IPROMS, Elsevier, Amsterdam, pp. 321-326.

Polygerinos, P., Wang, Z., Overvelde, J.T.B., Galloway, K.C., Wood, R.J., Bertoldi, K., Walsh, C.J., 2015. Modeling of Soft Fiber-Reinforced Bending Actuators. IEEE Trans. Robot. 31, 778-789. doi:10.1109/TRO.2015.2428504

RVK-Schunk [WWW Document], 2017. URL https://ch.schunk.com/ch_en/gripping-systems/ (accessed 6.1.17).

Salisbury, J.K., 1985. Kinematic and Force Analysis of Articulate Hands.

Salisbury, J.K., Craig, J.J., 1982. Articulated Hands: Force Control and Kinematic Issues. Int. J. Robot. Res. 1, 4-17. doi:10.1177/027836498200100102

SARAFun [WWW Document], 2017. URL http://h2020sarafun.eu/ (accessed 6.12.16).

Shadow Dexterous Hand, 2017.

Shepherd, R.F., Stokes, A.A., Nunes, R.M.D., Whitesides, G.M., 2013. Soft Machines That are Resistant to Puncture and That Self Seal. Adv. Mater. 25, 6709-6713. doi:10.1002/adma.201303175

Siciliano, B., Sciavicco, L., Villani, L., Oriolo, G., 2010. Robotics: Modelling, Planning and Control, 1st ed. 2009. ed. Springer, London.

Tai, K., El-Sayed, A.-R., Shahriari, M., Biglarbegian, M., Mahmud, S., 2016. State of the Art Robotic Grippers and Applications. Robotics 5, 11. doi:10.3390/robotics5020011

Velasco, J., V.B., Newman, W.S., 1998. Computer-assisted gripper and fixture customization using rapid-prototyping technology, in: 1998 IEEE International Conference on Robotics and Automation, 1998. Proceedings. Presented at the 1998 IEEE International Conference on Robotics and Automation, 1998. Proceedings, pp. 3658-3664 vol.4. doi:10.1109/ROBOT.1998.681393

Wang, Z., Hirai, S., 2017. Soft Gripper Dynamics Using a Line-Segment Model With an Optimization-Based Parameter Identification Method. IEEE Robot. Autom. Lett. 2, 624-631. doi:10.1109/LRA.2017.2650149 


\section{Appendix A: Index to multimedia Extensions}

\begin{tabular}{|l|l|l|}
\hline Extension & Media type & Description \\
\hline 1 & & $\begin{array}{l}\text { Performance experimental } \\
\text { verification video of: a) } \\
\text { Slot and Cylinder; b) Nut } \\
\text { and Bolt; and c) Concave } \\
\text { and Box. }\end{array}$ \\
\hline
\end{tabular}

